\title{
Hsp90 in the continuum of breast ductal carcinogenesis: Evaluation in precursors, preinvasive and ductal carcinoma lesions
}

\author{
Flora Zagouri1,2, Theodoros N Sergentanis ${ }^{1}$, Afrodite Nonni3 , Christos A Papadimitriou², Nikolaos V Michalopoulos' \\ Philip Domeyer' ${ }^{1}$, George Theodoropoulos ${ }^{1}$, Andreas Lazaris ${ }^{3}$, Effstratios Patsouris ${ }^{3}$, Eleni Zogafos ${ }^{1}$, Anastazia Pazaiti ${ }^{1}$ \\ and George C Zografos*1
}

\begin{abstract}
Background: Hsp90 (heat shock protein90) is a chaperone protein essential for preserving and regulating the function of various cellular proteins. Elevated Hsp90 expression seems to be a trait of breast cancer and may be an integral part of the coping mechanisms that cancer cells exhibit vis-à-vis stress. This manuscript tries to examine the immunohistochemical expression of Hsp90 all along the continuum of breast ductal lesions encompassing ductal hyperplasia without atypia (DHWithoutA), atypical ductal hyperplasia (ADH), ductal carcinoma in situ (DCIS) and invasive ductal carcinoma (IDC).
\end{abstract}

Methods: Tissue specimens were taken from 30 patients with DHWithoutA, 31 patients with ADH, 51 with DCIS and 51 with IDC. Immunohistochemical assessment of Hsp90 was performed both in the lesion and the adjacent normal breast ducts and lobules; the latter serving as control. Concerning Hsp90 assessment the percentage of positive cells and the intensity were separately analyzed. Subsequently, the Allred score was calculated. Post hoc analysis on the correlations between Hsp90 Allred score and possible predictors (grade, nodal status, tumor size, ER Allred score, PR Allred score, c-erbB-2 status and triple negative status) was conducted in IDC.

Results: Hsp90 exhibited mainly cytoplasmic immunoreactivity. Hsp90 Allred score exhibited an increasing trend along the continuum of breast ductal lesions (Spearman's rho $=0.169, p=0.031$ ). Compared to the adjacent normal ducts and lobules, no statistically significant differences were noted in DHwithoutA, ADH and DCIS. Hsp90 expression (intensity, positive cells, Allred score) was higher in IDC, compared to the adjacent normal tissue. Higher Hsp90 expression was observed in grade 2/3 IDCs (borderline association) and tumors of larger size. At the univariable analysis, higher Hsp90 expression was associated with higher ER Allred score, PR Allred score and c-erbB-2 positivity in IDC. Triple-negative IDCs exhibited significantly lower Hsp90 expression. The multivariable logistic regression model revealed that between the three markers, solely ER Allred score and c-erbB-2 positivity were independently associated with higher Hsp90 expression in IDC.

Conclusion: The above point to significant variability in Hsp90 expression with significant implications upon the effectiveness and limitations of anti-Hsp90 drugs.

\section{Background}

Hsp90 (heat shock protein 90, also known as HSPC1 according to the most recent nomenclature assigned by the HUGO Gene Nomenclature Committee [1]) is a

* Correspondence: gzografo@med.uoa.gr

1 Breast Unit, 1st Department of Propaedeutic Surgery, Hippokratio Hospital, School of Medicine, University of Athens, Greece

Full list of author information is available at the end of the article chaperone protein essential for preserving and regulating the function of various cellular proteins. Hsp90 mediates its actions through the formation of discrete subcomplexes, each containing proteins-cochaperones that assist protein folding and refolding during stress, protein transport and degradation [2-4]. Hsp90 interacts with a number of protein-cornerstones in breast carcinogenesis. Hsp90 counterparts include estrogen receptors (ER), p53 
protein, hypoxia-induced transcription factor HIF1alpha, protein kinase Akt, Raf-1 MAP kinase and a variety of receptor tyrosine kinases, such as erbB2 (reviewed in [5]).

Elevated Hsp90 expression seems to be a trait of breast cancer and may be an integral part of the coping mechanisms that cancer cells exhibit vis-à-vis stress [6-8]. As a result clinical implications of $\mathrm{Hsp} 90$ expression have emerged; Hsp90 upregulation may be a sign of unfavourable prognosis [9]. Another clinical implication concerns the pharmacological inhibition of Hsps [10-14]. Indeed, positive results have appeared in the literature concerning 17-allylamino, 17-demethoxygeldanamycin (17AAG), the first Hsp90 inhibitor having entered clinical trials $[5,15]$.

Previous studies have appeared concerning IDC $[9,16]$, where positive association between Hsp90 expression and grade, nodal positivity, tumour size, ER, c-erbB- 2 and decreased survival have been documented [9]. However, Hsp90 expression in precursor $(\mathrm{ADH})$ and preinvasive lesions (DCIS) have not yet been studied. Precursor and preinvasive lesions exhibit a distinct immunohistochemical profile regarding established markers such as ER, progesterone receptors (PR), c-erbB-2 etc [17]. It seems thus fairly rational to postulate that precursor and preinvasive lesions may also exhibit a distinct profile of Hsp90 expression. Interestingly, the sole study to focus exclusively on precursor lesions concerns the lobular series [18], where Hsp90 downregulation became unexpectedly evident.

Given the above, this is the first study to examine the immunohistochemical expression of Hsp90 all along the continuum of breast ductal lesions encompassing ductal hyperplasia without atypia (DHWithoutA), ADH, DCIS and IDC. In addition, a post hoc sub-analysis in IDC incorporating ER, PR, c-erbB-2 (integrated as triple negative status and assessed through a multivariable approach, for the first time to our knowledge), tumor size, grade and nodal status is presented.

\section{Methods}

This study included formalin-fixed, paraffin-embedded tissue specimens from 30 patients with DHWithoutA, 31 patients with ADH, 51 with DCIS and 51 patients with IDC. The patients' age at operation ranged between 31 and 78 (median age: 51 years). The diagnosis of the lesions was established by vacuum-assisted breast biopsy, excisional breast biopsy, lumpectomy and modified radical mastectomy. All lesions were independent i.e., no DHWithoutA, ADH, DCIS lesions coexisted with invasive carcinomas. Cases coexisting with lobular neoplasia and infiltrating lobular carcinoma were also excluded.

Hsp90 was immunohistochemically detected using the mouse monoclonal antibody Hsp90 (clone JPB24, NCL-
Hsp90, Novocastra supplied by Menarini). The dilution was 1:500 and the incubation time was equal to $18 \mathrm{~h}$ (at $4^{\circ} \mathrm{C}$ ). The visualization was performed using an avidinbiotin detection system $\mathrm{m}$ (Envision, Dako). Antigen retrieval was achieved in $0.01 \mathrm{M}$ citrate buffer $(\mathrm{pH}=6.0)$ at $85^{\circ} \mathrm{C}$ for $15 \mathrm{~min}$. Immunohistochemical assessment of Hsp90 was performed both in the lesion and the adjacent normal breast ducts and lobules; the latter serving as control. Negative controls were assessed by omitting the primary antibody.

Concerning Hsp90 assessment: i) the percentage of positive cells and ii) the intensity was separately analyzed. Subsequently, the Allred score was appropriately calculated summing the proportion score (represented by the estimated proportion of positive cells), as well as the intensity score (represented by the average intensity of positive cells) [19].

For immunohistochemistry (IHC), the following antibodies were used: PR (636, Dako), ER (ID5, Dako) and cerbB-2 (CB11, Novocastra $\left.{ }^{\mathrm{Tm}}\right)$. Sections ( $4 \mu \mathrm{m}$ thick) cut from formalin-fixed paraffin embedded tissue were used. After deparaffinization in xylene and hydration in graded ethanol solutions, the sections of IDC tissue were subjected to pretreatment in order to enhance antigen retrieval. The EnVision + System-HRP (DAB) (DakoCytomation, Glostrup, Danemark) was used with primary antibodies against the following antigens: PR, ER and cerbB-2. Immunohistochemistry was performed according to the protocols provided by the manufacturer. Concerning the immunohistochemical expression of ER and $\mathrm{PR}$, both the intensity (negative, $1+$ to $3+$ ) and percentage of immunopositive cells were evaluated. Subsequently, the Allred score was calculated [19].

The expression of c-erbB-2 was assessed as follows: i) negative, when no staining was documented or when membrane staining was present in less than $10 \%$ of tumor cells, ii) weak staining $(+)$, when partial membrane staining was documented in more than $10 \%$ of tumor cells, iii) moderate staining $(++)$ when weak/moderate complete membrane staining was present in more than $10 \%$ of tumor cells and iv) strong staining when strong, complete membrane staining was observed in more than $10 \%$ of tumor cells. Cases with negative and weak c-erbB-2 staining were considered as negative, whereas cases with strong c-erbB-2 staining were considered as positive. In cases with moderate staining, CISH (chromogenic in situ hybridization) was performed; subsequently these cases were considered as negative or positive. The ready-to-use Spot Light HER2/neu DNA Probe (Zymed/InVitrogen, San Fransisco, USA) was used for CISH; this digoxygenin-labeled probe is located on $17 \mathrm{q} 12-21$ and covers the entire gene area.

In all cases, ten fields ( $\times 40$ magnification) were assessed and a minimum of 100 cells were evaluated in the desig- 
nated areas, so as to evaluate the lesion as a whole. The immunohistochemical evaluation was performed independently by two consultant histopathologists (AN and AL).

The intensity was treated as an ordinal variable-score (0: negative, low: 1, moderate: 2, high: 3); Allred scores (ER, PR, Hsp90) have been treated as ordinal variables, whereas tumor size has been treated as a continuous variable. Due to deviation from the normal distribution, nonparametric statistics were chosen. The analysis included two steps: i) comparison of Hsp90 expression (Hsp90 intensity, positive cells (\%), Allred score) in the lesions $v s$. the adjacent normal ducts and lobules. Where appropriate, power calculations were performed. ii) A test for significance of the Hsp90 trend along the continuum of lesions. The correlation between Hsp90 Allred score in the lesion and the severity of the lesion (1:DHWithoutA, 2:ADH, 3:DCIS, 4:IDC) was evaluated; Spearman's rank correlation coefficient was appropriately implemented. iii) Post hoc analysis on the correlations between Hsp90 Allred score and possible predictors (grade, nodal status, tumor size, ER Allred score, PR Allred score, c-erbB-2 status and triple negative status) was conducted in IDC. Once again non-parametric statistics were performed under the light of deviation from normality and limited sample size. In addition, concerning the three immunohistochemical markers, multivariable logistic regression was performed as follows: Hsp90 Allred score was converted to a binary dependent variable [0: Hsp90 Allred score $\leq 7(n=28)$ and 1: Hsp90 Allred score $=8(n=23)$, as the median Hsp90 Allred score was equal to 7], whereas ER Allred score (continuous variable), PR Allred score (continuous variable) and c-erbB-2 status (positive $v s$. negative) were the independent variables.

Results of borderline statistical significance $(0.1<\mathrm{p}<$ 0.05) have also been reported, given that they might become significant in the context of a larger sample size. Statistical analysis was performed with STATA 8.0 statistical software (Stata Corporation, College Station, TX, USA).

Informed consent was obtained by all participants in this study. This study has been approved by the local Ethics Committee, in compliance to the Helsinki Declaration.

\section{Results}

Hsp90 exhibited mainly cytoplasmic immunoreactivity in epithelial cells of normal breast (ducts and lobules) (figure 1), ADH (figure 2), DCIS (figure 3) and IDC (figure 4, 5). Some epithelial cells also showed nuclear staining; nevertheless, all the DHWithoutA, ADH, DCIS and IDC foci mainly presented with a positive cytoplasmic immunoreaction for Hsp90. The percentage of these positive cells and the staining intensity were evaluated.

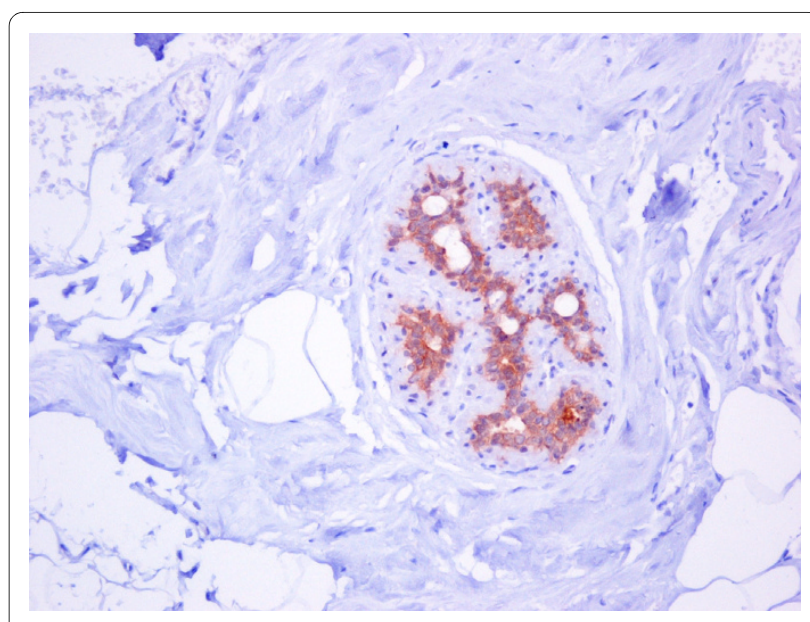

Figure 1 Moderate Hsp90 immunostaining in epithelial cells of normal breast (ducts and lobules) $(\times 200)$.

The percentage of Hsp90 positive cells, the intensity of Hsp90 staining, as well as the Allred score are presented in detail in Table 1. Compared to the adjacent normal ducts and lobules, no statistically significant differences were noted in DHWithoutA and ADH. Despite the numerical superiority in DCIS, the discrepancies failed to reach statistical significance. Indeed, given the present results, and assuming a type I error equal to 0.1 (threshold of borderline statistical significance), the study power was equal to 0.21 i.e. $21 \%$. Given the present results and under the same assumptions, 466 cases of DCIS would be needed to achieve the optimal study power of $80 \%$. Hsp90 expression (intensity, positive cells, Allred score) was higher in IDC, compared to the normal adjacent ducts and lobules (Table 1). Hsp90 Allred score exhibited an increasing trend along the continuum of breast ductal lesions (Spearman's rho $=0.169, \mathrm{p}=0.031$ )

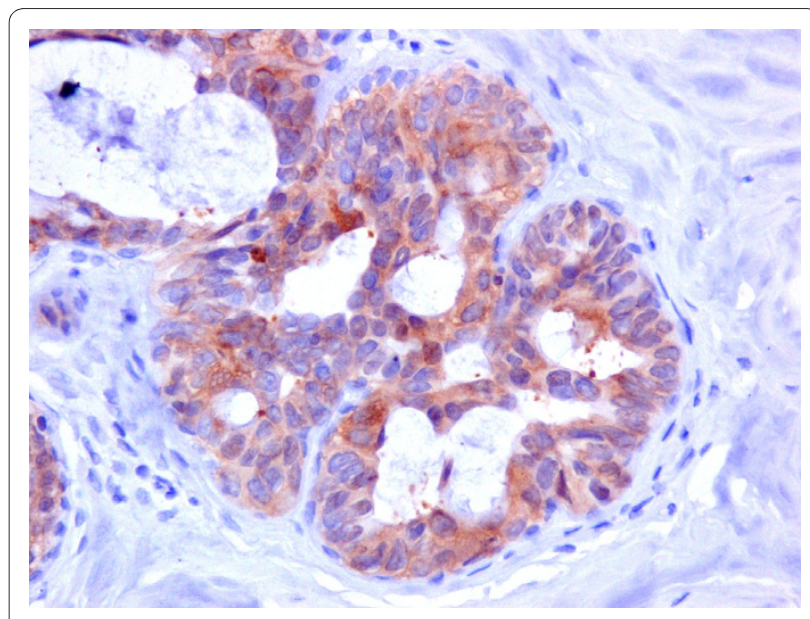

Figure 2 Moderate expression of $\mathrm{Hsp} 90$ in atypical ductal hyperplasia $(\times 400)$ 


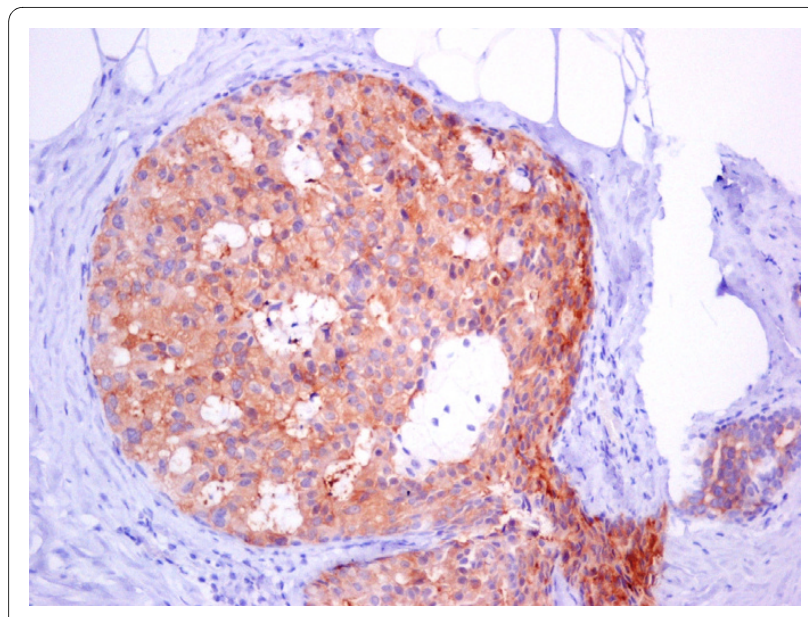

Figure 3 In situ ductal carcinoma with moderate expression of Hsp90 ( $\times 200)$

In the subanalysis concerning clinicopathological features (Table 2), higher Hsp90 expression was observed in grade 2/3 IDCs (borderline association), and larger tumor size. Table 2 presents the results in detail; median tumor size was equal to $2 \mathrm{~cm}$ and the dichotomization to cases $<$ median and $\geq$ median are provided for purely descriptive purposes, given that tumor size was treated as a continuous variable.

Table 3 presents the associations of Hsp90 Allred score with the immunohistochemical markers (figure 6, 7, 8). At the univariable analysis, higher Hsp90 expression was associated with higher ER Allred score (median: 7), PR Allred score (median:4) and c-erbB-2 positivity. Interestingly, triple-negative IDCs exhibited significantly lower Hsp90 expression (of note, the nodal status of triple-negative IDCs did not differ from the remaining tumors, $\mathrm{p}=$ 0.391, Mann-Whitney-Wilcoxon test for independent samples $[\mathrm{MWW}])$ present It is worth mentioning that ER

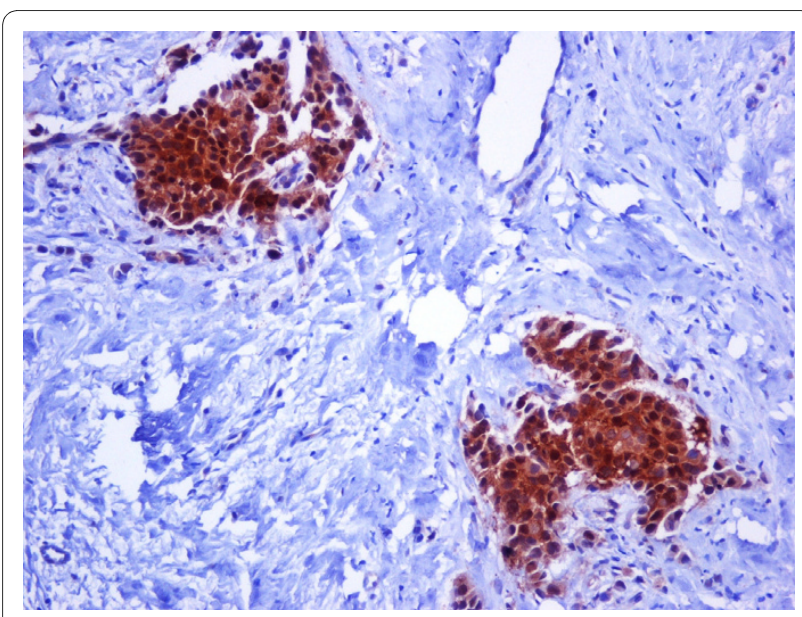

Figure 4 Intense expression of Hsp90 in invasive ductal carcinoma of the breast $(\times 200)$

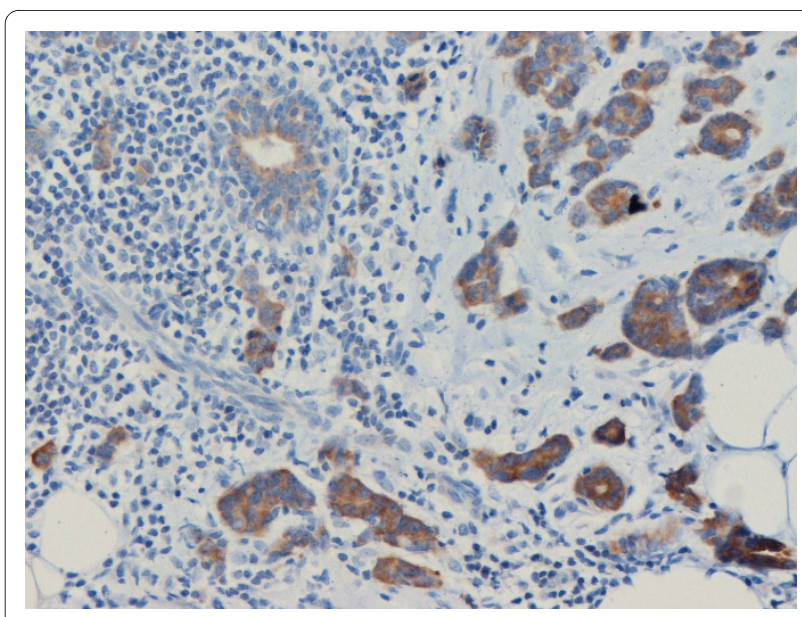

Figure 5 Both the percentage and the staining intensity are increased in invasive ductal carcinoma in comparison with the normal ductile $(\times 200)$

Allred score was positively associated with PR Allred score (Spearman's rho $=0.522, \mathrm{p}=0.0001$ ), whereas no significant association existed between ER Allred score and c-erbB-2 status ( $\mathrm{p}=0.849, \mathrm{MWW})$ nor between PR Allred score and c-erbB-2 status ( $\mathrm{p}=0.610$, MWW).

The multivariable logistic regression model revealed that between the three markers, solely ER Allred score $(\mathrm{OR}=1.29,95 \% \mathrm{CI}: 0.99-1.66)$ and c-erbB-2 positivity were independently associated with higher Hsp90 expression $(\mathrm{OR}=1.85$, 95\%CI: 1.14-3.02).

\section{Discussion}

This study is the first to investigate Hsp90 expression in precursor and preinvasive lesions. Noticeably, ADH did not exhibit a statistically significant difference whereas the numerical difference pointing to Hsp90 upregulation in DCIS failed to reach statistical significance. This finding may imply that the precursor context may not entail the cellular stress present in invasive cancer [18]. Consequently, as an integral part of the stress response, Hsp90 per se appears not to have been triggered early. Nevertheless, the steady numerical increase in Hsp90 Allred score along the ductal continuum may point to a simultaneous underlying increase in cellular stress, as well as to the multifaceted role mediated by Hsp90 in regulating the stability and function of many oncogenic and anti-oncogenic proteins, such as (ER), p53 protein, hypoxiainduced transcription factor HIF-1alpha, protein kinase Akt, Raf-1 MAP kinase and a variety of receptor tyrosine kinases, such as erbB2 [3-5].

At the end of the continuum, the gradual increase in Hsp90 expression reached statistical significance; Allred score and both its components (intensity and percentage of positive cells) were increased in IDC. Within the IDC group a variety of inherent tumour features seemed to 
Table 1: Hsp90 expression in ADH, DCIS and IDC

\begin{tabular}{|c|c|c|c|}
\hline & Adjacent normal ducts and lobules & DHwithoutA $(n=30)$ & $\mathbf{p}^{\mathbf{a}}$ \\
\hline Hsp90 intensity (score) & $2.27 \pm 0.58$ & $2.33 \pm 0.55$ & 0.593 \\
\hline Hsp90 positive cells (\%) & $70.7 \pm 9.44$ & $71.7 \pm 12.3$ & 0.664 \\
\hline \multirow[t]{2}{*}{ Hsp90 Allred score } & $7.00 \pm 0.87$ & $7.00 \pm 0.79$ & 0.896 \\
\hline & Adjacent normal ducts and lobules & $A D H(n=31)$ & $\mathbf{p}^{\mathbf{a}}$ \\
\hline Hsp90 intensity (score) & $2.26 \pm 0.63$ & $2.39 \pm 0.62$ & 0.346 \\
\hline Hsp90 positive cells (\%) & $69.4 \pm 10.3$ & $72.3 \pm 14.7$ & 0.195 \\
\hline \multirow[t]{2}{*}{ Hsp90 Allred score } & $6.97 \pm 0.98$ & $7.00 \pm 0.97$ & 0.660 \\
\hline & Adjacent normal ducts and lobules & DCIS $(n=51)$ & $\mathbf{p}^{\mathbf{a}}$ \\
\hline Hsp90 intensity (score) & $2.29 \pm 0.61$ & $2.43 \pm 0.67$ & 0.285 \\
\hline Hsp90 positive cells (\%) & $69.0 \pm 8.5$ & $71.0 \pm 14.9$ & 0.298 \\
\hline \multirow[t]{2}{*}{ Hsp90 Allred score } & $7.00 \pm 0.77$ & $7.14 \pm 0.94$ & 0.360 \\
\hline & Adjacent normal ducts and lobules & IDC $(n=51)$ & $\mathbf{p}^{\mathbf{a}}$ \\
\hline Hsp90 intensity (score) & $2.20 \pm 0.63$ & $2.49 \pm 0.54$ & 0.018 \\
\hline Hsp90 positive cells (\%) & $68.8 \pm 9.7$ & $74.3 \pm 14.9$ & 0.004 \\
\hline Hsp90 Allred score & $6.90 \pm 0.78$ & $7.33 \pm 0.77$ & 0.002 \\
\hline
\end{tabular}

ap-value derived from Wilcoxon matched-pairs signed-ranks test

correlate with higher Hsp90 expression. Tumors of larger size also presented with more pronounced Hsp90 upregulation, which is in accordance with previous reports [9] and seems to reflect the stress-related events in rapidly proliferating hypoxic (due to their size) tumours. Concerning tumor grade, however, the positive association with Hsp90 immunostaining should be interpreted with caution, given its borderline nature, as well as the small number of grade 1 IDCs in our sample.

Concerning immunohistochemical markers, this is the first study to adopt a multivariable approach. The univariable analysis shows that the higher Hsp90 expression was associated with higher ER, PR expression and c-erbB-2 positivity. The association between PR and Hsp90 expression is in line with previous studies [20,21]; nevertheless, given that ER and PR were closely associated in our study sample, as expected [22], this represented a secondary indirect association. As a result, the multivariable approach reported that the sole immunohistochemical markers independently associated with elevated Hsp90 expression are ER and c-erbB-2. This confirms and adds to the validity of the findings reported by Pick et al. [9], who however only performed a univariable analysis.

Commenting further on the immunohistochemical profile, significantly decreased Hsp90 expression has been observed in triple-negative tumours. This is in accordance with the study by Sun et al [16] and may seem fairly rational as Hsp90 is associated with ER and c-erbB2 expression. Our study highlights a paradox; on the one hand elevated Hsp90 has been suggested as a poor prognostic factor [9], whilst on the other hand triple-negative tumors, exhibiting decreased Hsp90 expression, are associated with poor prognosis (reviewed in [23]). Under the light of the above, the application of Hsp90 as a poor prognostic factor in all types of breast cancer may be worth interpreting with caution; accordingly, no statistically significant association emerged between nodal status and Hsp90 expression in our study, contrary to the report by Pick et al [9]. Nevertheless, the present study is not a prognosis study and thus no further firm conclu- 
Table 2: Subanalysis in IDC cases: clinicopathological variables

\begin{tabular}{|c|c|c|c|}
\hline Variables & N (\%) & Hsp90 Allred score in IDC & $\mathbf{p}$ \\
\hline Grade & & & $0.061^{\mathrm{Mww}}$ \\
\hline Grade 1 & $3(5.9 \%)$ & $6.67 \pm 0.58$ & \\
\hline Grade 2 \& 3 & $48(94.1 \%)$ & $7.38 \pm 0.76$ & \\
\hline Nodal status & & & $0.392^{\mathrm{S}}$ \\
\hline NO & 29 (56.9\%) & $7.28 \pm 0.84$ & \\
\hline N1 & $13(25.5 \%)$ & $7.23 \pm 0.73$ & \\
\hline N2 & $3(5.9 \%)$ & $7.67 \pm 0.58$ & \\
\hline N3 & $6(11.7 \%)$ & $7.67 \pm 0.52$ & \\
\hline Tumor size ${ }^{\S}$ & & & $0.033^{s}$ \\
\hline$<$ median & 20 (39.2\%) & $7.10 \pm 0.97$ & \\
\hline$\geq$ median & 31 (60.8\%) & $7.48 \pm 0.57$ & \\
\hline
\end{tabular}

sions can be drawn on this important field. Future studies focusing exclusively on triple negative tumors seem desirable, as triple-negative tumors were solely $9(17.7 \%)$ of all IDCs in this study.

The evaluation of Hsp90 expression all along the ductal continuum, presented herein, may have particular clinical significance. Firstly, given that ADH did not exhibit marked Hsp90 upregulation, the potential involvement of anti-Hsp90 drugs as chemoprevention agents may not be supported. In addition, given the associations between Hsp90, ER and c-erbB-2, it seems desirable that the effects of Hsp90 targeting drugs should be evaluated separately on different immunohistochemical types of IDC, since their effectiveness may vary accordingly.

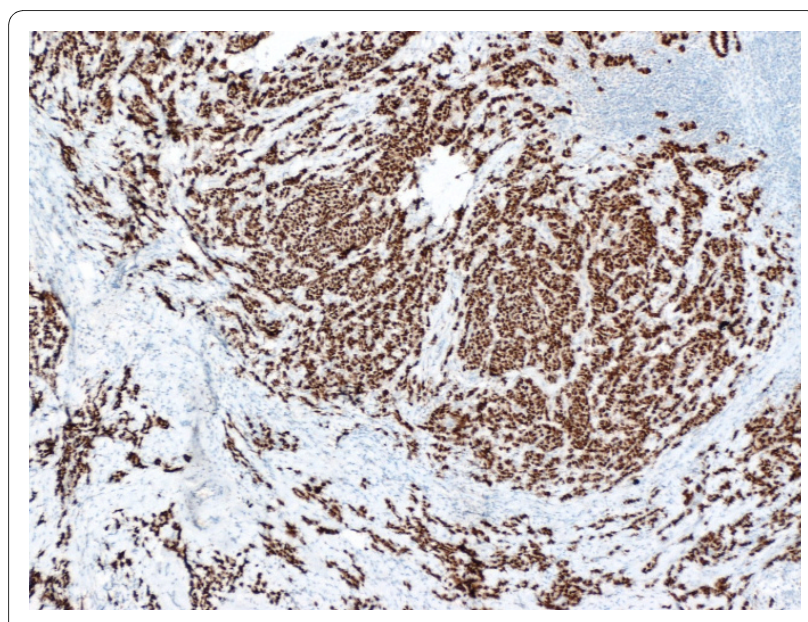

Figure 6 ER strong intensity in invasive ductal carcinoma of the breast $(\times 50)$.
In our cases, some epithelial cells of DHWithoutA, ADH, DCIS and IDC showed scarce nuclear Hsp90 localization. It should be declared that nuclear staining was not taken into account at the calculation of Allred score, as the latter was based exclusively on cytoplasmic Hsp90 staining. At any case, the significance of nuclear Hsp90 expression remains elusive, as some studies have not documented any nuclear Hsp90 expression in invasive ductal carcinomas [9], whereas other researchers have [24]. Other researchers have correlated nuclear Hsp90 staining with MHC class I expression in invasive breast carcinomas [25].

Concerning the limitations of this study, a number of statistical points should be noted. Firstly, although this study comprises the whole spectrum of ductal carcino-

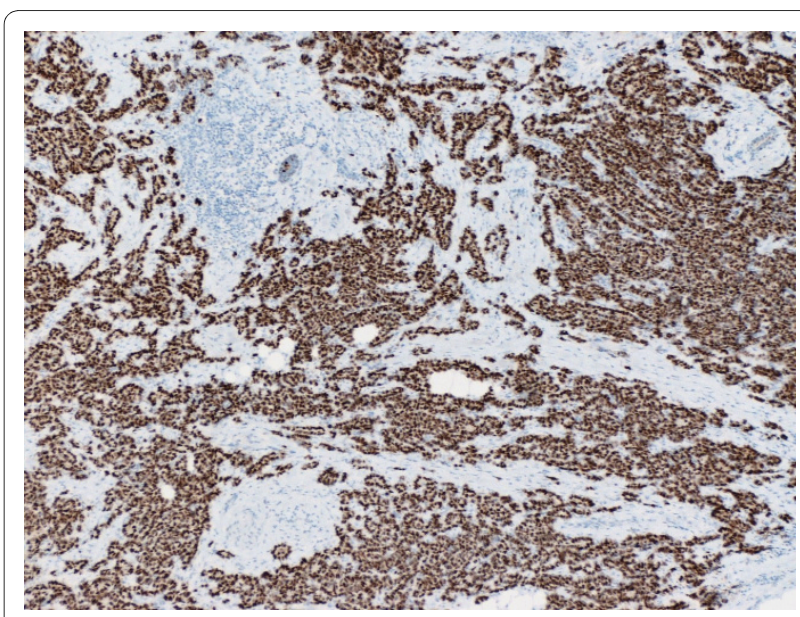

Figure 7 PR strong intensity in invasive ductal carcinoma of the breast $(\times 50)$. 


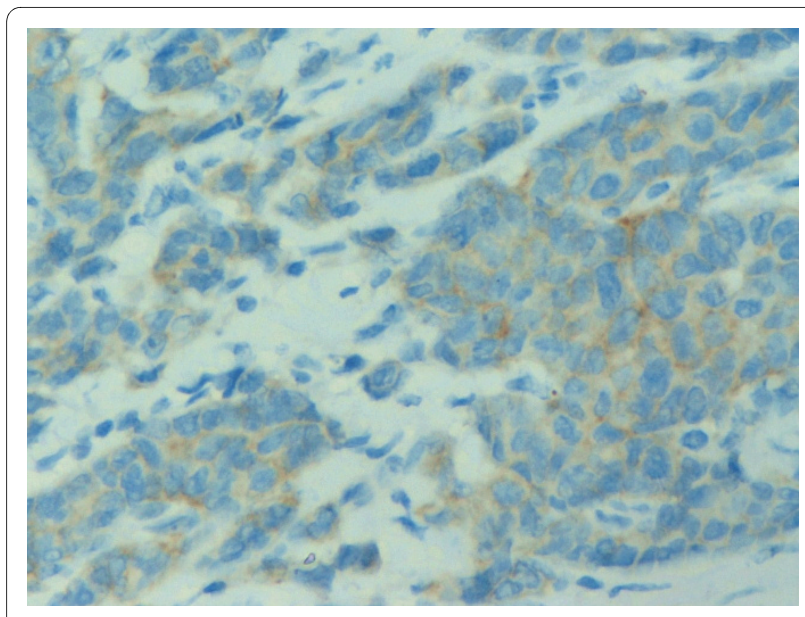

Figure 8 c-erbB2 no membranous staining in invasive ductal carcinoma of the breast $(\times 400)$. genesis, the lack of power may have inhibited the documentation of significant associations; for instance numerical discrepancies concerning DCIS may become significant in larger comparative studies (approximately 500 DCIS cases). Moreover, the limited sample size did not permit the performance of a multivariable analysis including both clinicopathological and immunohistochemical features, as the number of observations per cell was not sufficient. Nevertheless, this study is the first to adopt a multivariable assessment of Hsp90 predictors. On the other hand, despite the relatively small sample size, significant associations, such as the ER/Hsp90 and cerbB-2/Hsp90 interplay, have emerged and persisted at the multivariable approach; this may point to their reproducibility in larger samples. Nevertheless, further studies with larger number of patients are warranted.

Other limitations of this study pertain to the method performed (i.e., immunohistochemistry); the lack of an

Table 3: Subanalysis in IDC cases: immunohistochemical variables

\begin{tabular}{|c|c|c|c|}
\hline \multicolumn{4}{|c|}{ Univariable analysis } \\
\hline Variables & N (\%) & Hsp90 Allred score & $\mathbf{p}$ \\
\hline Triple-negative status ${ }^{\S}$ & & & $0.0004^{\mathrm{MwW}}$ \\
\hline Triple-negative & $9(17.7 \%)$ & $6.44 \pm 1.01$ & \\
\hline Non-triple-negative & $42(82.3 \%)$ & $7.52 \pm 0.55$ & \\
\hline ER Allred score ${ }^{\dagger}$ & & & $0.004^{s}$ \\
\hline$<$ median & $23(45.1 \%)$ & $7.04 \pm 0.93$ & \\
\hline$\geq$ median & $28(54.9 \%)$ & $7.57 \pm 0.50$ & \\
\hline PR Allred score ${ }^{\dagger}$ & & & $0.020^{\mathrm{s}}$ \\
\hline$<$ median & $21(41.2 \%)$ & $6.95 \pm 0.86$ & \\
\hline$\geq$ median & $30(58.8 \%)$ & $7.60 \pm 0.56$ & \\
\hline c-erbB-2 status & & & $0.011 \mathrm{Mww}$ \\
\hline Negative & $29(56.9 \%)$ & $7.10 \pm 0.86$ & \\
\hline Positive & $22(43.1 \%)$ & $7.64 \pm 0.49$ & \\
\hline
\end{tabular}

Multivariable analysis

\begin{tabular}{lccc}
\hline Variables & Category or increment & OR (95\% Cl) & p \\
\hline ER Allred score & 1 point increase ${ }^{\ddagger}$ & $1.29(0.99-1.66)$ & $\mathbf{0 . 0 5 5}$ \\
PR Allred score & 1 point increase & $1.18(0.92-1.51)$ & 0.195 \\
C-erbB-2 status & Positive vs. negative & $1.85(1.14-3.02)$ & $\mathbf{0 . 0 1 3}$ \\
\hline
\end{tabular}

${ }_{M W W} \mathrm{p}$-value derived from Mann-Whitney-Wilcoxon test for independent samples; ${ }^{5} p$-value derived from Spearman's rank correlation coefficient; ${ }^{\S}$ not included in the multivariable analysis, as it integrates all three components (ER, PR, c-erbB-2); ${ }^{+}$ER Allred score, PR Allred score have been treated as continuous variables and thus the subcategories serve purely descriptive purposes ${ }^{\ddagger}$ practical interpretation: increase in Allred score by one unit predicts $\mathrm{OR}=1.29$. 
automated quantitative procedure may have clouded some associations. To ensure the objectivity of the assessment, the percentage and intensity were assigned by two independent pathologists blind to one another's results; however, the need of additional verification of the present results through Western blot should be declared. Moreover, all comparisons have been made versus the adjacent normal ducts and lobules, as previously described [18]; it would be of interest to verify the present results also on normal glands taken for example from reduction mammoplasties or adjacent to fibroadenomas. In addition, future studies should examine whether the findings of the subanalysis on IDC (i.e., those implicating ER, PR and cerb-B2) are reproducible in DHWithoutA, ADH or DCIS; data on these subpopulations were not available in this study. Finally, reporting the survival would yield additional information concerning the clinical significance of Hsp90 expression; the design of this retrospective study did not include follow-up for survival analysis.

\section{Conclusion}

In conclusion, DHWithoutA, ADH and DCIS do not exhibit marked Hsp90 upregulation, while IDC present with elevated Hsp90 expression. Within IDC, a higher grade, larger tumor size, higher ER expression and cerbB-2 positivity correlated with higher Hsp90 expression. Noticeably, triple-negative tumours exhibited decreased Hsp90 expression. The above point to significant variability in Hsp90 expression with significant implications upon the effectiveness and limitations of anti-Hsp90 drugs.

\section{Competing interests}

The authors declare that they have no competing interests.

\begin{abstract}
Authors' contributions
All authors read and approved the final manuscript.

FZ: conceived the idea, participated in the design of the study and assisted in the writing of the manuscript; TNS: participated in the design of the study, performed the statistical analysis and assisted in the writing of the manuscript; AN: performed the pathological evaluation; CAP: evaluated critically the manuscript; NVM: performed vacuum-assisted breast biopsy and open surgery; PD: performed vacuum-assisted breast biopsy and open surgery; GT: performed vacuum-assisted breast biopsy and open surgery; AL: performed the pathological evaluation; EP: evaluated critically the manuscript; EZ: revised and evaluated critically the manuscript; AP: performed vacuum-assisted breast biopsy and open surgery, evaluated critically the manuscript; GCZ: conceived of the study, participated in its design, performed vacuum-assisted breast biopsy and open surgery and evaluated critically the manuscript
\end{abstract}

\section{Acknowledgements}

We would like to thank the President of the Hellenic Anticancer Institute Dr. Efstathios Fragoulis, for the technical organization of the Breast Unit and we would like to thank the Special Account for Research Grants of the University of Athens for the financial support. No conflict of interests exists.

\section{Author Details}

'Breast Unit, 1st Department of Propaedeutic Surgery, Hippokratio Hospital, School of Medicine, University of Athens, Greece, ${ }^{2}$ Department of Clinical Therapeutics, Alexandra Hospital, School of Medicine, University of Athens, Greece and ${ }^{31}$ st Department of Pathology, School of Medicine, University of Athens, Greece
Received: 26 December 2009 Accepted: 5 July 2010

Published: 5 July 2010

\section{References}

1. Kampinga HH, Hageman J, Vos MJ, Kubota H, Tanguay RM, Bruford EA Cheetham ME, Chen B, Hightower LE: Guidelines for the nomenclature of the human heat shock proteins. Cell Stress Chaperones 2009, 14:105-111.

2. Whitley D, Goldberg SP, Jordan WD: Heat shock proteins: a review of the molecular chaperones. J Vasc Surg 1999, 29:748-751.

3. Whitesell L, Lindquist SL: HSP90 and the chaperoning of cancer. Nat ReV Cancer 2005, 5:761-72.

4. Zuehlke A, Johnson $\mathrm{J}$ : $\mathrm{Hsp} 90$ and co-chaperones twist the functions of diverse client proteins. Biopolymers 2010, 93:211-7.

5. Beliakoff J, Whitesell L: Hsp90: an emerging target for breast cancer therapy. Anticancer Drugs 2004, 15:651-662.

6. Conroy SE, Latchman DS: Do heat shock proteins have a role in breast cancer? Br J Cancer 1996, 74:717-721.

7. Yano M, Naito Z, Tanaka S, Asano G: Expression and roles of heat shock proteins in human breast cancer. Jpn J Cancer Res 1996, 87:908-915.

8. Yano M, Naito Z, Yokoyama M, Shiraki Y, Ishiwata T, Inokuchi M, Asano G: Expression of hsp90 and cyclin D1 in human breast cancer. Cancer Lett 1999, 137:45-51.

9. Pick E, Kluger Y, Giltnane JM, Moeder C, Camp RL, Rimm DL, Kluger HM: High HSP90 expression is associated with decreased survival in breast cancer. Cancer Res 2007, 67:2932-2937.

10. Kim LS, Lee HS, Choi JW, Kang HJ, Price JE: The role of heat shock protein $90 / 70$ as potential molecular therapeutic targets in breast cancer. In Proceedings of the 90th annual meeting of the American Association for Cancer Research 2005 April 16-20, Anaheim, CA Philadelphia, PA: AACR; 2005. Abstract no. 2346

11. Miyata Y: Hsp90 inhibitor geldanamycin and its derivatives as novel cancer chemotherapeutic agents. Curr Pharm Des 2005, 11:1131-1138.

12. Bagatell $\mathrm{R}$, Whitesell $\mathrm{L}$ : Altered $\mathrm{Hsp} 90$ function in cancer: a unique therapeutic opportunity. Mol Cancer Ther 2004, 3:1021-1030.

13. Sôti C, Nagy E, Giricz Z, Vígh L, Csermely P, Ferdinandy P: Heat shock proteins as emerging therapeutic targets. Br J Pharmacol 2005, 146:769-780.

14. Workman P, Burrows F, Neckers L, Rosen N: Drugging the cancer chaperone HSP90: combinatorial therapeutic exploitation of oncogene addiction and tumor stress. Ann N Y Acad Sci 2007, 1113:202-216

15. Holmes JL, Sharp SY, Hobbs S, Workman P: Silencing of HSP90 cochaperone AHA1 expression decreases client protein activation and increases cellular sensitivity to the HSP90 inhibitor 17-allylamino-17demethoxygeldanamycin. Cancer Res 2008, 68:1188-1197.

16. Sun $B$, Zhang $S$, Zhang D: Identification of metastasis-related proteins and their clinical relevance to triple-negative human breast cancer. Clin Cancer Res 2008, 14:7050-9.

17. Zagouri F, Sergentanis TN, Zografos GC: Precursors and preinvasive lesions of the breast: the role of molecular prognostic markers in the diagnostic and therapeutic dilemma. World J Surg Oncol 2007, 5:57.

18. Zagouri F, Nonni A, Sergentanis TN, Papadimitriou CA, Michalopoulos NV, Lazaris AC, Patsouris E, Zografos GC: Heat shock protein90 in lobular neoplasia of the breast. BMC Cancer 2008, 8:312.

19. Allred DC, Harvey JM, Berardo M, Clark GM: Prognostic and predictive factors in breast cancer by immunohistochemical analysis. Mod Pathol 1998, 11:155-168.

20. Shyamala G, Schweitzer M, Ullrich SJ: Relationship between 90kilodalton heat shock protein, estrogen receptor, and progesterone receptor in human mammary tumors. Breast Cancer Res Treat 1993 26:95-100.

21. Lemoisson E, Cren H, Goussard J: Chromatographic separation of eight progesterone receptor isoforms in human breast tumors, and detection by radioligand and monoclonal antibodies. Association with hsp90 and hsp70 heat shock proteins. Ann Biol Clin (Paris) 1994, 52:433-42.

22. O'Malley FP, Peder SE: In Invasive carcinomas: Special types in Breast Pathology. Elsevier 2006:204.

23. Reis-Filho JS, Tutt AN: Triple negative tumours: a critical review. Histopathology 2008, 52:108-18. 
24. Diehl MC, Idowu MO, Kimmelshue K, York TP, Elmore LW, Holt SE: Elevated expression of nuclear Hsp90 in invasive breast tumors. Cancer Biol Ther 2009, 8:1952-61.

25. Gebhard B, Schütz G, Ecker RC, Steiner GE, Rudas M, Gnant M, Oehler R: MHC-class-I expression in human breast cancer correlates with nuclear localization of the $90 \mathrm{kDa}$ heat-shock-protein. Anticancer Res 1999, 19:5293-5297.

Pre-publication history

The pre-publication history for this paper can be accessed here: http://www.biomedcentral.com/1471-2407/10/353/prepub

doi: $10.1186 / 1471-2407-10-353$

Cite this article as: Zagouri et al., Hsp90 in the continuum of breast ductal carcinogenesis: Evaluation in precursors, preinvasive and ductal carcinoma lesions BMC Cancer 2010, 10:353

Submit your next manuscript to BioMed Centra and take full advantage of:

- Convenient online submission

- Thorough peer review

- No space constraints or color figure charges

- Immediate publication on acceptance

- Inclusion in PubMed, CAS, Scopus and Google Scholar

- Research which is freely available for redistribution

Submit your manuscript at www.biomedcentral.com/submit 